\title{
The Mars thermosphere-ionosphere: Predictions for the arrival of Planet-B
}

\author{
S. W. Bougher ${ }^{1}$ and H. Shinagawa ${ }^{2}$ \\ ${ }^{1}$ Lunar and Planetary Laboratory, University of Arizona, Tucson, AZ 85721, U.S.A. \\ ${ }^{2}$ Solar Terrestrial Environment Laboratory, Nagoya University, Japan \\ (Received July 28, 1997; Revised January 6, 1998; Accepted February 12, 1998)
}

\begin{abstract}
The primary science objective of the Planet-B mission to Mars is to study the Martian upper atmosphere-ionosphere system and its interaction with the solar wind. An improved knowledge of the Martian magnetic field (whether it is induced or intrinsic) is needed, and will be provided by Planet-B. In addition, a proper characterization of the neutral thermosphere structure is essential to place the various plasma observations in context. The Neutral Mass Spectrometer (NMS) onboard Planet-B will provide the required neutral density information over the altitude range of $150-500 \mathrm{~km}$. Much can be learned in advance of Planet-B data taking as multi-dimensional thermosphere-ionosphere and MHD models are exercised to predict the Mars near-space environment that might be expected during the solar maximum conditions of Cycle 23 (1999-2001). Global model simulations of the Mars thermosphere-ionosphere system are presented and analyzed in this paper. These Mars predictions pertain to the time of Planet-B arrival in October 1999 (F10.7 200; Ls 220). In particular, the National Center for Atmospheric Research (NCAR) Mars Thermosphere General Circulation Model (MTGCM) is exercised to calculate thermospheric neutral densities $\left(\mathrm{CO}_{2}\right.$, $\left.\mathrm{CO}, \mathrm{N}_{2}, \mathrm{O}, \mathrm{Ar}, \mathrm{O}_{2}\right)$, photochemical ions $\left(\mathrm{CO}_{2}^{+}, \mathrm{O}_{2}^{+}, \mathrm{O}^{+}\right.$below $\left.200 \mathrm{~km}\right)$, neutral temperatures, and 3-components winds over 70-300 km. Cases are run with and without dust loading of the lower atmosphere in order to examine the potential impacts of dust storms on the thermosphere-ionosphere structure. Significant dust-driven impacts are predicted in the lower thermosphere $(100-120 \mathrm{~km})$, but are less pronounced above $150 \mathrm{~km}$. The ionospheric peak height changes greatly with the passage of a Mars global dust storm event. In addition, Martian dayside exobase temperatures are generally warmer during dusty periods, in accord with Mariner 9 UVS data (Stewart et al., 1972). During the Planet-B mission, the NMS team intends to use the MTGCM as a facility tool whose simulated output can be utilized to aid various investigations.
\end{abstract}

\section{Background and Motivation}

The Mars thermosphere is the region of the upper atmosphere above $\sim 100 \mathrm{~km}$, where the temperature increases with increasing altitude. The Martian thermospheric structure is generally thought to be controlled by solar EUV and UV processes (like Venus), since Mars may have a rather small intrinsic magnetic field. Solar cycle, seasonal, and orbital variations of the Mars upper atmosphere are indeed expected and generally observed (Stewart and Hanson, 1982; Stewart, 1987; Bougher et al., 1990; Barth et al., 1992). Missions at or near solar minimum (Mariner 4, Viking) have yielded dayside temperatures (140-240 K) that are about a factor of 1.5-2.0 smaller than temperatures (260-350 K) obtained by missions at or near solar maximum (Mariner 6, 7 and 9) (see Barth et al., 1992). Nightside thermospheric observations are presently lacking. Solar forcing, although usually dominant, is not always the primary driver of the current Mars upper atmosphere (Stewart et al., 1972, 1992; Bougher et al., 1993). Available data suggest a significant forcing of the thermosphere from below as a result of upward propagating gravity waves and/or tides. These forcings are episodic in nature, changing greatly with season and with the occurrence of planetwide dust storms. Mariner 9 UVS data clearly imply significant warming of Mars dayside temperatures due

Copy right (C) The Society of Geomagnetism and Earth, Planetary and Space Sciences (SGEPSS); The Seismological Society of Japan; The Volcanological Society of Japan; The Geodetic Society of Japan; The Japanese Society for Planetary Sciences. to wave processes operating during the 1971-72 global dust storm (Stewart et al., 1972, 1992). The interaction of these gravity waves/tides with the EUV-UV solar-driven Mars thermospheric structure and dynamics is important to quantify in order to accurately assess the present behavior of the Martian thermosphere (Bougher et al., 1993, 1997). Subsequently, this neutral thermospheric structure provides the seed for corresponding ion production and photochemical ion distributions over the dayside $(\leq 200 \mathrm{~km})$. Predictions of future thermosphere-ionosphere conditions at Mars are thus complicated by the uncertain role of these temporal forcings arising from dust storm events (Bougher et al., 1997).

Planet-B is slated to make detailed measurements of the Mars neutral and plasma environment during the upcoming solar maximum period of cycle 23 (1999-2001). The 150 $\mathrm{km}$ by $15 R_{\mathrm{M}}$ Planet-B orbit will be inclined $170^{\circ}$ to the ecliptic plane, with a period of $\sim 34$ hours (Yamamoto and Tsuruda, 1998, this issue). The latitude of periapsis will scan the entire low-latitude band in a Martian year because of the $24^{\circ}$ Mars obliquity. This orbit, essentially fixed in inertial space as Mars revolves around the sun, is quite similar to that of Pioneer Venus (Colin, 1979). A Neutral Mass Spectrometer (NMS) onboard Planet-B will measure various neutral densities, and will provide temperature profiles derived from neutral scale heights. This in-situ data (at equatorial latitudes) will be complemented by Ultraviolet Spectrometer (UVS) airglow data yielding neutral densities and 
temperatures at other latitudes and local times than those near periapsis. These two instruments will characterize the thermospheric structure during this solar maximum period. The NMS and UVS neutral data will also serve as a basic resource for nearly all other plasma experiments on the spacecraft.

The primary science objective of the Planet-B mission is to study the Martian upper atmosphere-ionosphere system and its interaction with the solar wind. In addition, measurements of key neutral constituents are vital to our understanding of the processes that maintain the structure, energetics, and dynamics of the Mars upper atmosphere, and the processes that contribute to atmospheric escape and the evolution of Mars' water and climate. These two goals are interdependent, in that the characterization of present atmospheric processes is necessary to extrapolate escape processes into the past. The NMS and UVS are two of many Planet-B instruments whose data will be assimilated to address these secondary science objectives. Finally, comparative studies of Venus, Earth, and Mars neutral upper atmospheres can be launched in earnest with the advent of this new Planet-B NMS and UVS database.

Multi-dimensional modeling tools are needed both to predict what might be expected upon Planet-B arrival at Mars, and to systematically analyze the Planet-B neutral and plasma datasets that will be obtained. The National Center for Atmospheric Research (NCAR) Mars Thermosphere General Circulation Model (MTGCM) is one such model that specifically addresses the thermospheric composition, energetics, and dynamics over 70-300 km (see Section 2). Feedback of energetic, dynamic, photochemical, and diffusive processes is addressed by this 3-D framework. The MTGCM code can be utilized to: (a) extract the dynamical and energetic processes that maintain the observed structure of the Mars upper atmosphere, (b) engage in collaborative studies to extract the escape processes responsible for present day atmospheric loss from Mars, and (c) extrapolate these escape processes into the past in order to examine the evolution of Mars water and climate history. The MTGCM code is presently being exercised to compile a library of MTGCM simulations for various solar, orbital, seasonal, and dust conditions likely to be encountered by Planet-B during NMS data taking. This archive, covering periods of the Planet-B nominal mission, will provide the framework for initial NMS investigations once neutral density data is collected and analysis begins. Later, during the Planet-B prime mission, the MTGCM code will be run for actual Mars conditions and its output made available as a data analysis tool for scientific investigations by other Planet-B teams (see Section 5).

The primary goals of this paper are twofold: (a) to illustrate the capability of the MTGCM code and its usefulness in Planet-B data analysis and subsequent collaborative studies among various Planet-B teams, and (b) to provide a prediction of the structure of the Mars thermosphere-ionosphere upon Planet-B arrival at Mars in October 1999. A basic description of the MTGCM code and its relevant inputs are described in Section 2. MTGCM modeling results for October 1999 are described and analyzed in Section 3; cases with and without a dusty lower Mars atmosphere are presented. Section 4 discusses the implications of these MTGCM simulations for subsequent magnetohydrodynamic (MHD) model simulations. Finally, we close with a summary and a brief report of our plan to make MTGCM output available to the Planet-B community at large.

\section{Three-Dimensional Modeling Tools and Inputs 2.1 MTGCM hydrodynamic model}

Over the past 10-years, the NCAR terrestrial Thermosphere-Ionosphere General Circulation Model (TIGCM) (Roble et al., 1988) has been adapted to the unique fundamental parameters, physics, and inputs appropriate to the Mars upper atmosphere (Bougher et al., 1988, 1990, 1993; Fox et al., 1995; Bougher, 1995; Bougher et al., 1997). This Mars Thermospheric General Circulation Model (MTGCM) has been successfully used to address the upper atmospheric structure and dynamics above $\sim 70 \mathrm{~km}$. The MTGCM has been validated using Mariner 9 Ultraviolet Spectrometer

(UVS) data that provide dayside oxygen mixing ratios near the ionospheric peak (Stewart et al., 1992). Also, several airglow measurements by various spacecraft (Mariner 6, 7, 9) and descent probe density profiles (Viking 1 and 2, Mars Pathfinder) provide scale heights and inferred temperatures for constraining MTGCM simulations (e.g. Stewart et al., 1972; Nier and McElroy, 1977; Seiff and Kirk, 1977; Barth et al., 1992; Schofield et al., 1997). Solar cycle, seasonal, and orbital behavior of the Mars upper atmosphere has been predicted using this MTGCM model. In recent years, the MTGCM has been modified to examine the impact of dustdriven atmospheric inflation and enhanced semi-diurnal tides upon the upper atmospheric structure and dynamics (Bougher et al., 1993, 1997); refinement of the Mars Global Surveyor aerobraking strategy was made as a result of this modeling activity (Bougher et al., 1997). It is clear that the MTGCM is a very useful tool for: (1) predicting the Mars upper atmosphere environment for future spacecraft operations, (2) initially characterizing the Mars structure and dynamics for future data analysis, and (3) unraveling the feedback mechanisms and processes that maintain and drive variations in the upper atmosphere structure and wind system. It is also possible for the MTGCM to be configured with enhanced solar EUV fluxes so as to simulate Mars upper atmosphere conditions at previous solar epochs (Bougher and Fox, 1996).

The code itself is a finite-difference primitive equation model that self-consistently solves for steady-state or timedependent neutral temperatures, neutral-ion densities, and 3 -component neutral winds over the globe. The model is both a predictive and diagnostic tool, capable of extracting the balances maintaining the observed structure and dynamics (and variations) of the Martian mesosphere and thermosphere (Bougher et al., 1990, 1993). The physical processes incorporated into the model are those appropriate to thermospheric dynamics; i.e. fast molecular vertical diffusion of heat, momentum, and constituents at thermospheric heights (e.g. Roble et al., 1988). Most importantly, the MTGCM is firmly based on well studied $\mathrm{CO}_{2}$ energetic and chemical processes for Venus that should apply to Mars as well (Bougher, 1995). Prognostic equations for the major neutral species $\left(\mathrm{CO}_{2}, \mathrm{CO}, \mathrm{N}_{2}\right.$, and $\left.\mathrm{O}\right)$, selected minor neutral species (Ar and $\mathrm{O}_{2}$ ), and several photochemical ions (e.g. $\mathrm{O}_{2}^{+}, \mathrm{CO}_{2}^{+}, \mathrm{O}^{+}$ below $200 \mathrm{~km}$ ) are included. Zonal, meridional, and vertical velocities, total temperatures, and geopotential heights are 
also obtained on 33-pressure levels (above 1.32 micro-bar), corresponding to $\sim 70-300 \mathrm{~km}$, with a $5^{\circ}$ latitude and longitude resolution. The vertical coordinate is log-pressure, with a vertical spacing of two grid points per scale height.

Adjustable parameters which can be varied for individual MTGCM cases include the F10.7-cm index (solar EUV-UV flux variation), heliocentric distance (orbital variation), solar declination (seasonal variation), and the maximum eddy coefficient $\left(K_{\mathrm{t}}\right)$ for eddy diffusion and viscosity. This eddy coefficient is prescribed to be roughly a factor of 3 smaller than presently used in one-dimensional models of the Martian upper atmosphere (e.g. Krasnopolsky, 1993). This reflects the mixing effect of large scale winds in the MTGCM, and the corresponding reduced requirement for eddy mixing. The MTGCM can also be modified to accommodate atmospheric inflation and semi-diurnal $(2,2)$ to $(2,6)$ tidal mode amplitudes and phases consistent with dusty conditions present in the Mars lower atmosphere during global dust storm events (Bougher et al., 1993, 1997).

\subsection{MTGCM input parameters for October 1999}

The MTGCM parameters prescribed for this Planet-B simulation correspond to Mars conditions for October 8, 1999, which is roughly the time of Planet-B orbit insertion. At this time: (a) the Mars heliocentric distance is $1.398 \mathrm{AU}$, (b) the Mars season is Southern Spring $\left(L_{\mathrm{s}}=220^{\circ}\right)$, and (c) the solar declination is $-16.2^{\circ}$ latitude. Current estimates for solar activity (Joselyn et al., 1997; Schatten and Pesnell, 1993) during the Planet-B mission suggest solar maximum conditions, with correspondingly higher temperatures and densities than observed for solar minimum Viking data. We adopt a mean F10.7-cm index of 200 units to approximate the solar maximum EUV-UV fluxes needed, using reference flux data sets of Torr et al. $(1979,1980)$ and Torr and Torr (1985). These parameters yield a scaled Martian F10.7-cm flux of 102 units, which is quite similar to that for previous Mariner 6-7 observations. These scaled EUV-UV fluxes change dramatically over the course of the Mars year, due to the large orbital eccentricity of the planet. The resulting variations in thermospheric structure and dynamics are predicted to be significant (see Bougher et al., 1990). Corresponding variations in photochemical ions are also expected.

Reasonable MTGCM parameters are used for Mars thermospheric heating and cooling: (a) the EUV-UV heating efficiency utilized is 20-22\% (Fox and Dalgarno, 1979); (b) the $\mathrm{CO}_{2}-\mathrm{O}$ relaxation rate is chosen to be $1.5 \times 10^{-12} \mathrm{~cm}^{3} / \mathrm{sec}$ (Bougher et al., 1994); and (c) the rather uncertain eddy coefficient for diffusion and conduction is purposely held to the presently estimated value of $1.5 \times 10^{7} \mathrm{~cm}^{2} / \mathrm{sec}$. The MTGCM height scale is calibrated to NASA Ames GCM lower atmosphere model simulations (Murphy et al., 1995; Bougher et al., 1997; see Subsection 2.3) for identical Mars season and dust-free conditions. Electron and ion temperatures are empirically prescribed in a manner that reproduces their presently observed behavior up through the ionopause, and allows for some variation with solar fluxes (see Fox, 1993). Ion temperatures are prescribed to be equal to neutral temperatures below $180 \mathrm{~km}$; above $300 \mathrm{~km}$, the ion temperature is set to the electron temperature; in between, a smoothly varying matching formulation is used. This behavior approximates the ion temperatures measured by the RPA on Viking, as presented by Hanson et al. (1977). The assumed electron temperature profile is similar to that calculated by Rohrbaugh et al. (1979). These assumptions are sufficient for our MTGCM simulations, since the relevant recombination rates and charge exchange reactions affecting dayside photochemical ions (below $200 \mathrm{~km}$ ) are only weakly temperature dependent. Most key neutral-ion reactions and rates employed in the MTGCM formulation are identical to those used by Fox (1993) and Fox et al. (1995).

\subsection{Dusty lower atmosphere inputs}

Available spacecraft and ground-based data are presently insufficient to characterize the response of the Mars atmosphere $(0-200 \mathrm{~km})$ to dust storm events. However, coupled simulations of Mars lower and upper atmospheric models can be used to estimate the general response of the Mars atmosphere to various background mean dust levels and/or simulated dust storm events that might occur during the course of a Martian year (Bougher et al., 1997). Two key mechanisms have been identified that regulate this dust-driven coupling of the Mars lower and upper atmospheres: (1) atmospheric inflation due to aerosol heating, and (2) the generation, propagation, and dissipation of semidiurnal tides that significantly impact the $100-120 \mathrm{~km}$ region. Gravity waves may also modulate the tidal impacts predicted. In particular, such waves may induce both momentum deposition and eddy diffusion. The former will decelerate the wind velocity if the waves are generated by surface topographic features. Conversely, gravity waves may accelerate the flow if they are generated by dust storms. Eddy diffusion enhanced by gravity-wave breaking may also contribute to dissipation of tidal waves.

Two general circulation models are currently being run in tandem to investigate the impact of inflation and semidiurnal tides upon the Mars upper atmosphere. The Mars lower atmosphere model chosen is the NASA Ames Mars General Circulation Model (MGCM), a primitive equation, grid-point numerical model of the Martian atmosphere (0$100 \mathrm{~km})$. It contains a variety of numerical parameterizations for the treatment of such physical processes as radiative transfer (solar absorption and infrared absorption and emission by gaseous $\mathrm{CO}_{2}$ and suspended dust), atmospheric/surface interactions (transfer of momentum and sensible heat), condensation/sublimation of carbon dioxide (and the concurrent changes in atmospheric mass), and imposed flow deceleration near the model top (for both physical and numerical reasons). The MGCM is presently exercised with a horizontal resolution of $7.5^{\circ}$ in latitude and $9^{\circ}$ in longitude. Vertically, the model consists of 26 layers extending from the surface to a pressure of 0.2 micro-bar, which is near $100 \mathrm{~km}$. The reliability of this MGCM code in reproducing the actual Martian lower atmosphere climate and weather systems is complicated by: (a) un-predictable dust storm events and their time- and spatially-variable dust opacities, (b) non-LTE IR heating and cooling above $80 \mathrm{~km}$, and (c) gravity wave breaking processes. These factors will modify the solution resulting from the coupled lower and upper atmosphere models (see Subsection 3.3). The Ames MGCM is thoroughly discussed in Pollack et al. (1990) and Murphy et al. (1995).

In the present Mars model coupling scheme, the Ames MGCM is first run for specified Mars seasonal conditions for which a constant background dust level is imposed and 
the model heating and winds are allowed to self-consistently evolve. (Alternatively, a source region and impulse function can be specified for lofting dust from the MGCM surface; thereafter, the MGCM code is exercised to allow dust to be transported around the planet self-consistently, while radiative effects of this dust feed back into the energetics and dynamics.) The coupling of the two Mars GCM codes is subsequently accomplished by passing zonally averaged mean temperatures and heights from the MGCM to the MTGCM at the 1.32 micro-bar level (the MTGCM lower boundary). This provides a connection between the lower-upper atmosphere codes which accounts for the thermal expansion and contraction of the lower atmosphere with the evolution of a dust storm. In addition, the MTGCM lower boundary heights and semi-diurnal tidal amplitudes and phases are extracted from the same constant pressure surface in the Ames MGCM simulation (see details given in Bougher et al., 1993, 1997). These semi-diurnal tidal fields being passed between these GCM codes represent only a portion of the total wave component present in the MGCM at the 1.32 micro-bar level, thus neglecting any gravity wave modification of tidal effects. This is a necessary simplification at this stage of model development (see Section 5).

For the purpose of illustrating the potential behavior of the Mars upper atmosphere during dusty conditions, a Planet$\mathrm{B}$ case is run corresponding to a dusty lower atmosphere with a spatially averaged, static visible dust opacity of 1.0. This might correspond to dust conditions just prior to the 1977a dust storm that Viking-1 experienced. The resultant MGCM 1.32 micro-bar semi-diurnal tidal mode amplitudes (up to $\pm 1.2 \mathrm{~km}$ ) and phases are specified at the MTGCM lower boundary at the same level. The corresponding MTGCM lower boundary heights (79 to $63 \mathrm{~km}$ from South pole to North pole) are also specified according to MGCM output. Notable differences between the dust-free simulated fields (see Subsection 3.1) and these dust-driven fields are described in Subsection 3.2.

\section{MTGCM Modeling Results}

\subsection{Planet-B MOI conditions: No dust}

MTGCM (benchmark) simulations are presented for F10.7 = 102 units at Mars, corresponding to expected Mars conditions during Planet-B orbit insertion around October 8, 1999. No dust-driven effects are simulated for this MTGCM case. However, the heights at 1.32 micro-bar are taken from the Ames MGCM at the same pressure surface for this season. We examine the basic characteristics of the simulated Mars neutral thermosphere and photochemical ionosphere.

Figure 1(a) is a latitude vs. local time slice of temperatures and horizontal winds near the MTGCM (benchmark) exobase at $205 \mathrm{~km}$. Temperatures peak at $333 \mathrm{~K}$ in the Southern polar day, and are a minimum at $136 \mathrm{~K}$ in the Northern polar night. Mid-afternoon temperatures $(\mathrm{LT}=15)$ at the subsolar latitude reach about 310-320 K, near the lower limit of those estimated from Mariner 6-7 airglow observations (315-350 K) (see Barth et al., 1992). This large day-night temperature gradient gives rise to a pressure gradient which drives global winds which diverge from a point in the midlatitude Southern hemisphere (near LT $=15$ ) and converge near the morning terminator in the mid-latitude Northern hemisphere. Also, winds are seen to converge near LT $=20$ at the equator; a corresponding "heat island" is generated at this location resulting from adiabatic compressional heating. Horizontal winds grow to as large as $310 \mathrm{~m} / \mathrm{s}$ over the terminators and in the Northern polar night. Overall, the global wind and thermal structure is indicative of in-situ solar EUVUV heating, and the feedback of winds upon the thermal structure is very important (see Bougher et al., 1990).

Figure 1(b) shows a similar latitude vs. local time slice of temperatures and horizontal winds, now taken from the homopause region at $125 \mathrm{~km}$. This surface is just above the mesopause, where temperatures begin to rise rapidly with increasing altitude. Such a transition corresponds to the growing importance of solar EUV-UV over solar IR heating. The day-night temperature gradient at the homopause level is now about $80 \mathrm{~K}$, reduced from $\sim 200 \mathrm{~K}$ at the exobase. Appropriately reduced winds blow away from the mid-latitude Southern hemisphere (near LT $=15$ ) and converge near the morning terminator in the mid-latitude Northern hemisphere. In addition, evidence of the "heat island" (arising from adiabatic heating) still remains at LT $=20-21$. Polar night temperatures drop to $127 \mathrm{~K}$; this suggests that a nearly isothermal thermosphere exists in the polar night region. Dayside temperatures peak at just over $200 \mathrm{~K}$ in the Southern subtropics; the vertical temperature gradient between 100 and $220 \mathrm{~km}$ in this region is large $(170 \mathrm{~K})$ (see Fig. 2(a)). Horizontal winds grow to $235 \mathrm{~m} / \mathrm{s}$ across the poles. Features resulting from solar EUV-UV-IR heating are modified by the feedback of winds upon the thermal structure. This feedback drives a "quasi-semidiurnal" signature in the simulated temperature field.

Figure 2(a) illustrates MTGCM (benchmark) equatorial temperatures over all local times from 100 to $300 \mathrm{~km}$. Notice that nightside contours do not extend up to $300 \mathrm{~km}$; this reflects the fact that the MTGCM constant pressure surfaces are closer together in these colder regions of the Mars nightside. As stated above, dayside exospheric temperature values $(310 \mathrm{~K})$ are in the lower range of those inferred from Mariner 6-7 airglow data. Overall, temperatures are fairly isothermal everywhere above $210-220 \mathrm{~km}$. The vertical temperature gradient is large $(170 \mathrm{~K})$ in the mid-afternoon $(\mathrm{LT}=15)$, while it is quite small $(70 \mathrm{~K})$ in the early morning $(\mathrm{LT}=4)$. The corresponding diurnal temperature variation at exospheric heights $(\geq 205 \mathrm{~km})$ is about $110-120 \mathrm{~K}$. This simulated diurnal contrast is about half of that observed in the Venus upper atmosphere (200 K) (cf. Bougher, 1995). This result suggests that the Mars rotation precludes any effective isolation of the day and nightsides, thereby reducing the expected diurnal thermal constrast from that observed at Venus (see Bougher et al., 1990; Bougher, 1995). Recently obtained Mars Pathfinder (MPF) density and inferred temperatures over $0-160 \mathrm{~km}$ (LT $=3-4$ and $19^{\circ} \mathrm{N}$. latitude) are presently being analyzed (Schofield et al., 1997). However, these entry measurements are probably unsuitable to constrain the MTGCM nightside thermospheric structure above $125 \mathrm{~km}$.

Figure 2(b) illustrates MTGCM (benchmark) equatorial $\mathrm{O} / \mathrm{CO}_{2}$ mixing ratios over all local times from 100 to 240 $\mathrm{km}$. The dayside $(\mathrm{LT}=15)$ value near the ionospheric peak $(\sim 125 \mathrm{~km})$ (see Fig. $2(\mathrm{c})$ ) is just under $2 \%$. Atomic oxygen 
MTGCM NEUTRAL TEMPERATURE (DEG K)

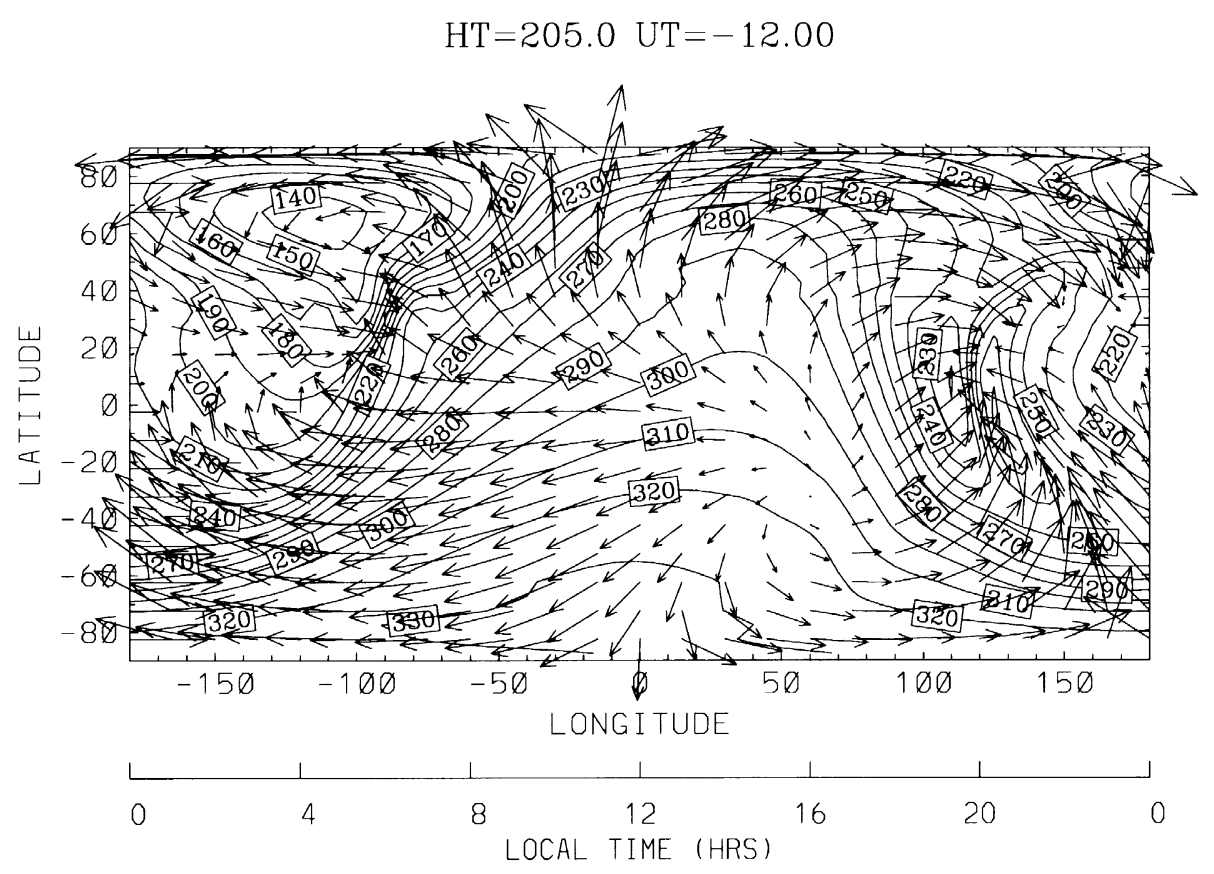

(a)

MTGCM NEUTRAL TEMPERATURE (DEG K)

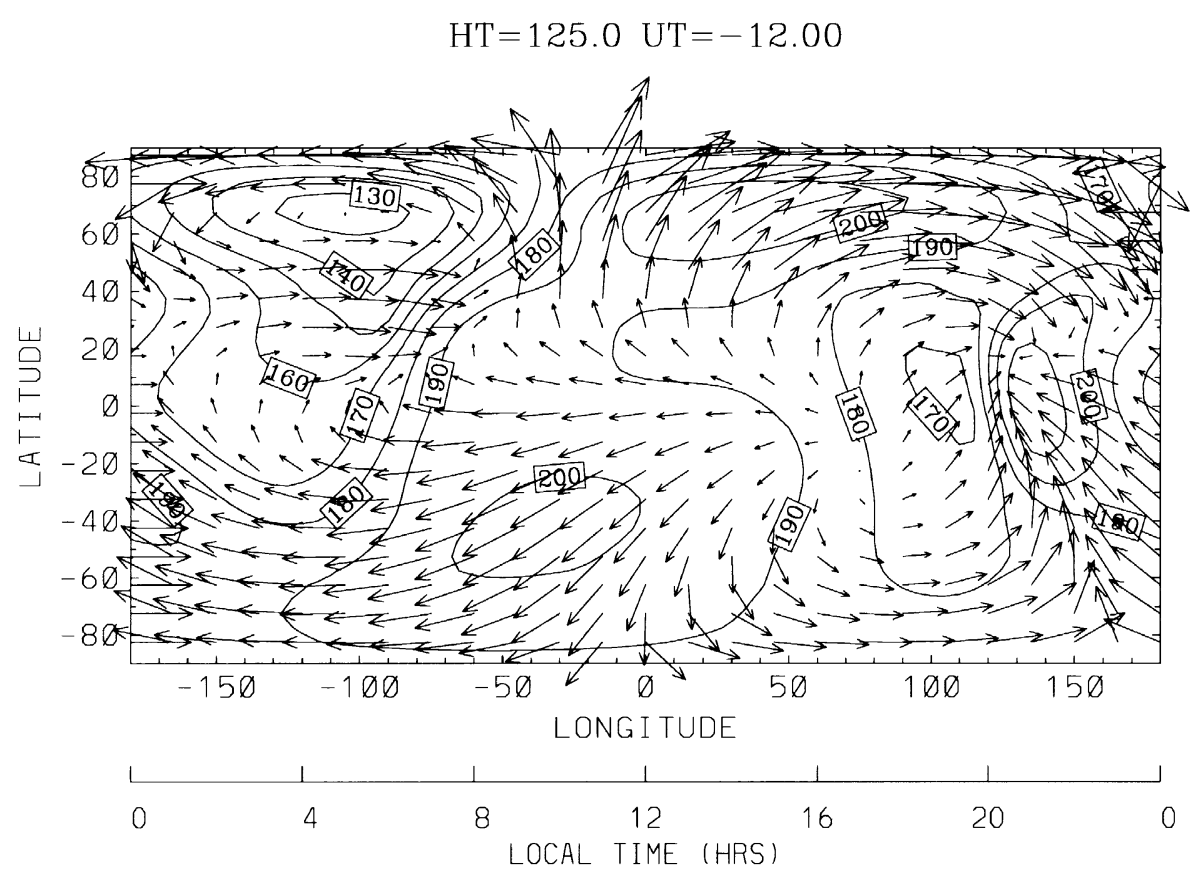

(b)

Fig. 1. Planet-B MOI MTGCM simulation (F10.7 = 102 at Mars; no dust): (a) Constant altitude slice (latitude vs. longitude or local time) at the exobase $(\sim 205 \mathrm{~km})$ for $T$ (total temperature) $+(u, v)$ (horizontal winds). Temperature extremes range from 135.6 to $332.7 \mathrm{~K}$. The length of the maximum arrow represents $310 \mathrm{~m} \mathrm{sec}^{-1}$. (b) Constant altitude slice (latitude vs. longitude or local time) at the homopause $(\sim 125 \mathrm{~km})$ for $T$ (total temperature) $+(u, v)$ (horizontal winds). Temperature extremes range from 126.5 to $216.8 \mathrm{~K}$. The length of the maximum arrow represents $235 \mathrm{~m} \mathrm{sec}^{-1}$. 


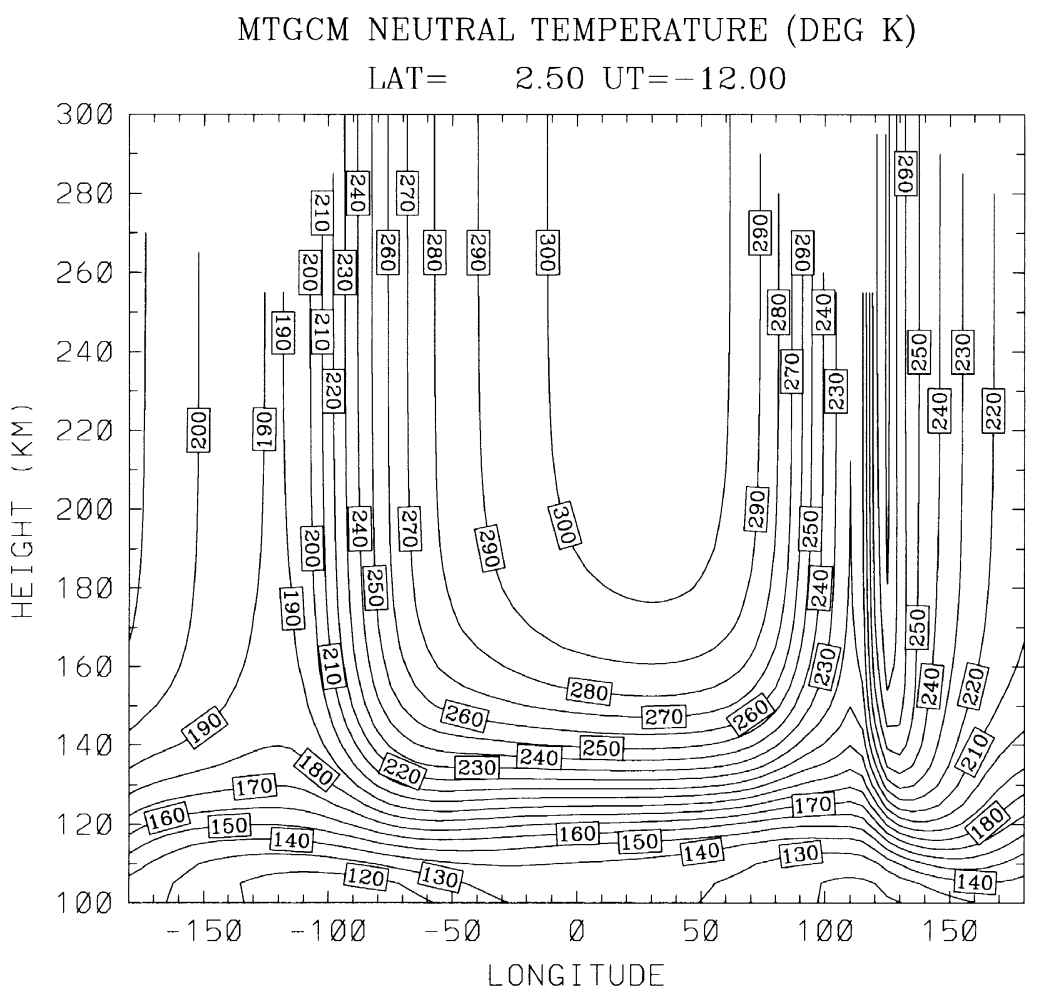

(a)

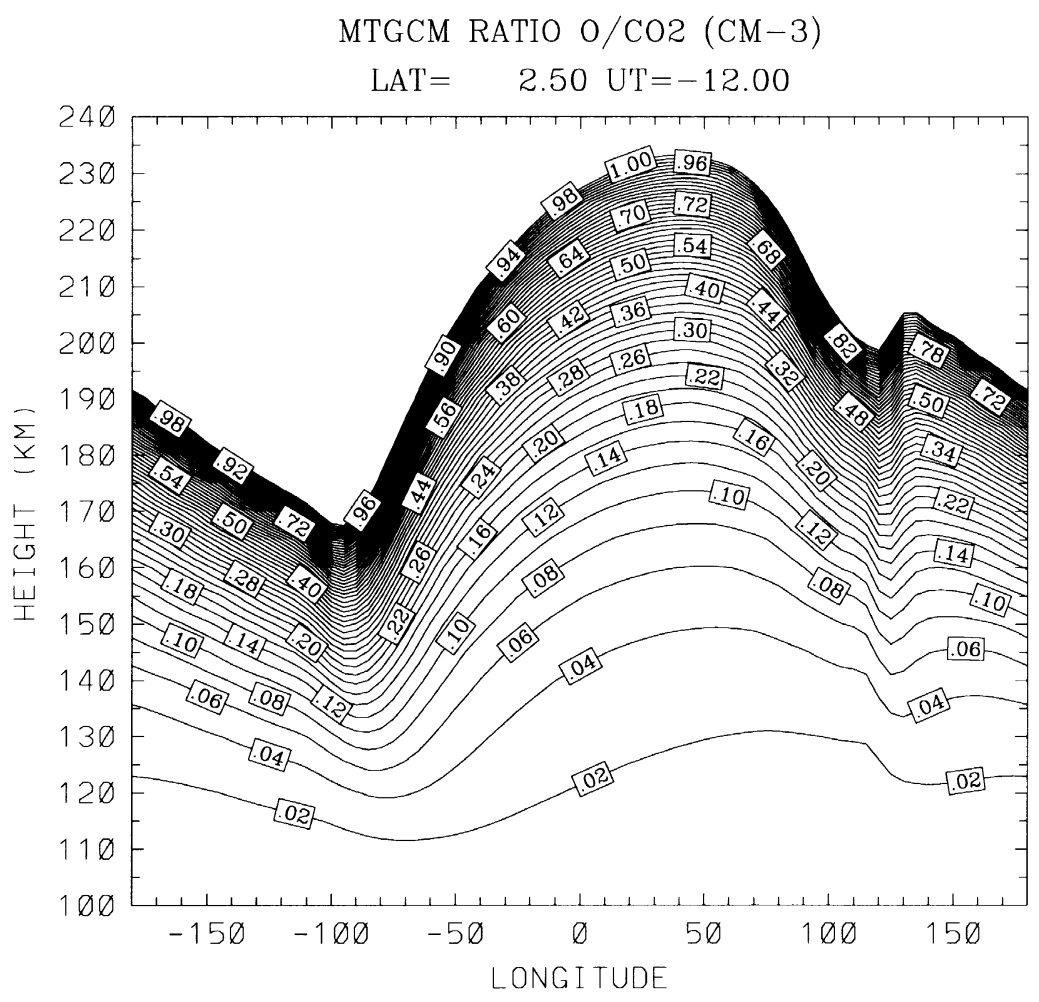

(b)

Fig. 2. Planet-B MOI MTGCM simulation (F10.7 = 102 at Mars; no dust): (a) $2.5 \mathrm{~N}$ latitude slice of total temperature over 100-300 km, with extremes ranging from 112 to $306.3 \mathrm{~K}$; (b) $2.5 \mathrm{~N}$ latitude slice of $\mathrm{O} / \mathrm{CO}_{2}$ mixing ratio over $100-240 \mathrm{~km}$, with extremes ranging from 0.02 to 1.0 ; saturation above 1.0 is shown; (c) Constant LT $=1500$ (altitude vs. latitude) slice of electron density ( $\log 10)$ over 110-200 km with extremes ranging from $1.0 \times 10^{4}$ to $2.0 \times 10^{5} \mathrm{~cm}^{-3}$. The longitude scales given in (a) and (b) can be converted to local time by referring to the $x$-axis scales of Fig. 1 . 


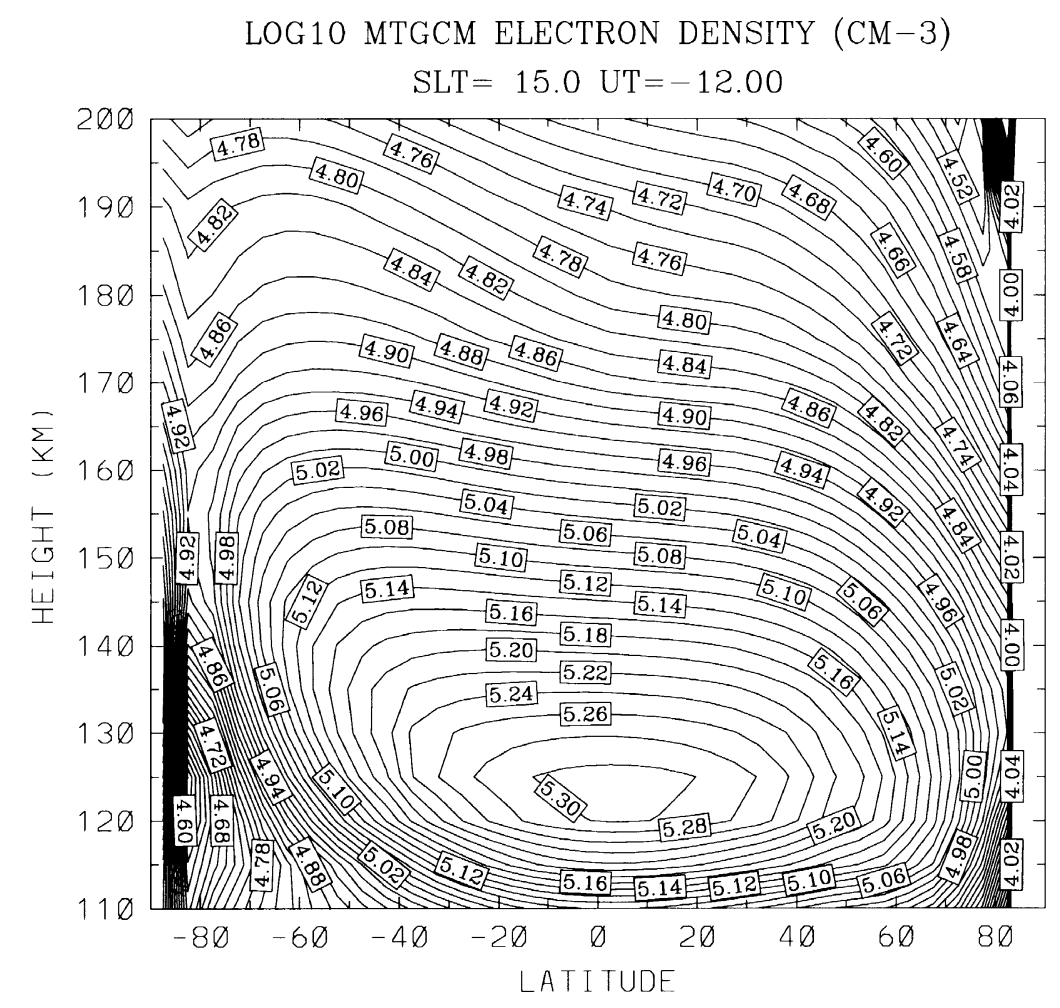

(c)

Fig. 2. (continued).

exceeds $\mathrm{CO}_{2}$ on the dayside above about $220-230 \mathrm{~km}$; this level drops significantly onto the nightside (down to $170 \mathrm{~km}$ at LT $=4$ ), in accord with colder nightside temperatures and smaller scale heights. The dayside ionospheric peak value is consistent with the spread of $\mathrm{O} / \mathrm{CO}_{2}$ values estimated from various proxy methods (1.25\% during Viking and 2-3\% during Mariner 6-7) (see discussion in Bougher et al., 1990). The simulated diurnal variation of oxygen suggests that the wind system transports oxygen atoms efficiently away from their dayside source (Stewart et al., 1992).

Figure 2(c) shows a constant local time slice $(\mathrm{LT}=15)$ of MTGCM (benchmark) simulated electron densities over $110-200 \mathrm{~km}$. Recall that photochemical equilibrium has been assumed in this region for the MTGCM formulation (see Subsection 2.1). Terminator effects have been minimized in this plot by setting $1.0 \times 10^{4} \mathrm{~cm}^{-3}$ as the lower limit contour level. An electron density peak of $2 \times 10^{5}$ $\mathrm{cm}^{-3}$ occurs at $\sim 125 \mathrm{~km}$ near the equator $\left(\sim 45^{\circ} \mathrm{SZA}\right)$, while a peak of $1.4 \times 10^{5} \mathrm{~cm}^{-3}$ occurs at $135 \mathrm{~km}$ near $60^{\circ} \mathrm{S}$ latitude $\left(\sim 60^{\circ} \mathrm{SZA}\right)$. This rise of the peak altitude for increasing SZA under relatively constant Southern hemisphere temperatures is expected from theory. Also, this electron peak value at $60^{\circ} \mathrm{SZA}$ compares favorably with that calculated previously $\left(\sim 1.75 \times 10^{5} \mathrm{~cm}^{-3}\right)$ by Fox et al. (1995) for solar maximum conditions. Another test of the reliability of the simulated dayside $\mathrm{O} / \mathrm{CO}_{2}$ mixing ratio is the calculated value of the $\mathrm{O}_{2}^{+}$to $\mathrm{CO}_{2}^{+}$ratio near the ionospheric peak. The MTGCM (benchmark) value for $\mathrm{O}_{2}^{+} / \mathrm{CO}_{2}^{+}$(not shown) of $\sim 3.5$ at $45^{\circ} \mathrm{SZA}$ is about half that observed by the Viking
RPA (6.0-7.0) at $45^{\circ} \mathrm{SZA}$. This suggests that our O-mixing ratios are slightly too small for these solar maximum conditions. Larger O-mixing ratios can be obtained by using the improved solar EUV fluxes of Tobiska (1991), especially shortward of $250 \mathrm{~A}$, which will also enhance the magnitude of the electron density at the peak (see Fox et al., 1995). In general, we have confidence that our photochemical ion scheme is realistic near the ionospheric peak region. Above $220 \mathrm{~km}$, this assumption will not be appropriate for $\mathrm{O}^{+}$, due to the role of ambipolar diffusion. Furthermore, solar-wind induced horizontal advection of ions may be important above $\sim 200 \mathrm{~km}$ (Shinagawa and Cravens, 1989). Nevertheless, this MTGCM formulation for photochemical ions is a necessary precursor to a global simulation of ions that incorporates ion drift velocities under specific magnetic conditions. See Section 4 for more discussion.

Figures 3(a) and 3(b) illustrate MTGCM (benchmark) equatorial density profiles at: (a) $\mathrm{LT}=15$ (dayside), and (b) $\mathrm{LT}=5$ (nightside). $\mathrm{CO}_{2}, \mathrm{CO}, \mathrm{N}_{2}, \mathrm{O}, \mathrm{Ar}$, and $\mathrm{O}_{2}$ densities are plotted. The dayside profiles are not unlike those estimated previously for Mariner 6-7 conditions (see Bougher et al., 1990; Fox et al., 1995). The nightside profiles are pure speculation, since no neutral thermosphere data presently exist to provide a constraint.

\subsection{Planet-B MOI conditions: With dust}

A dusty lower atmosphere is simulated to explore the potential impacts upon the Martian thermosphere and ionosphere during the Planet-B MOI period. A static background of spatially uniform (horizontal) dust is assumed with a vis- 


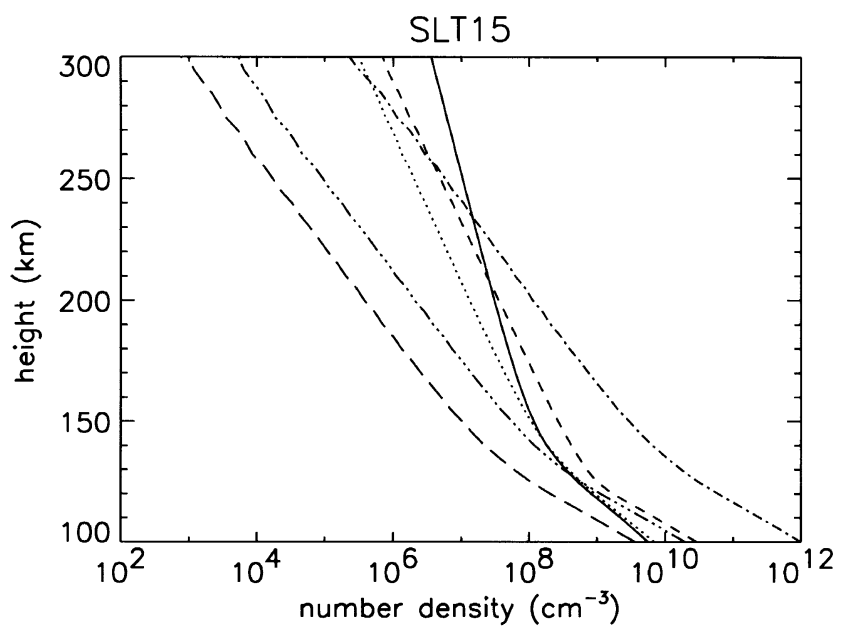

(a)

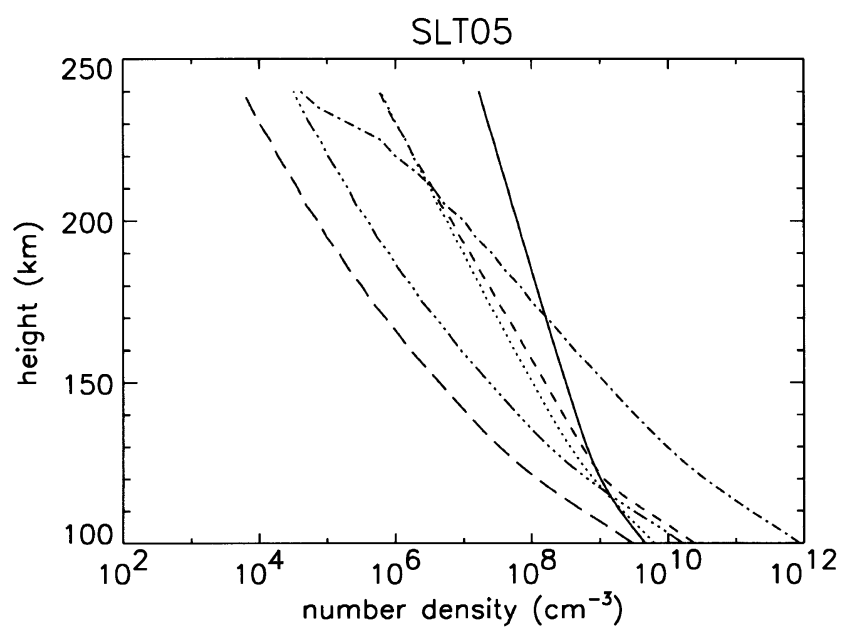

(b)

Fig. 3. Planet-B MOI MTGCM simulation (F10.7 = 102 at Mars; no dust): (a) Dayside $\mathrm{LT}=1500$ profiles of neutral densities at $2.5 \mathrm{~N}$ latitude $\left(\mathrm{CO}_{2}\right.$, $\mathrm{CO}, \mathrm{N}_{2}, \mathrm{O}, \mathrm{Ar}, \mathrm{O}_{2}$ ) spanning 100-300 km; (b) Nightside LT $=0500$ profiles of neutral densities at $2.5 \mathrm{~N}$ latitude $\left(\mathrm{CO}_{2}, \mathrm{CO}, \mathrm{N}_{2}, \mathrm{O}, \mathrm{Ar}, \mathrm{O}_{2}\right)$ spanning 100-240 km. The curves represent these densities as follows: $\mathrm{O}(-), \mathrm{CO}(\cdots), \mathrm{N}_{2}(---), \mathrm{CO}_{2}(-\cdot), \mathrm{Ar}(-\cdots)$, and $\mathrm{O}_{2}(--)$.

ible optical depth of 1.0. This MTGCM (dusty) simulation requires inputs from the NASA Ames GCM (see Subsection 2.3 above for details). Presently, gravity wave fluxes are not being transferred from the lower atmosphere (MGCM) into the thermosphere (MTGCM).

Figure 4(a) is a latitude vs. local time slice of temperatures and horizontal winds near the MTGCM (dusty) exobase at $205 \mathrm{~km}$. This plot is to be compared with that of Fig. 1(a) to assess dust impacts. Temperatures now peak at $363 \mathrm{~K}$ near the equator at LT $=14-15$, and are a minimum at $152 \mathrm{~K}$ also near the equator at LT $=22$. These mid-afternoon temperatures $(\mathrm{LT}=15)$ are nearly $50-60 \mathrm{~K}$ warmer than the MTGCM (benchmark) case. Such a warming is consistent with Mariner 9 dayside temperatures that were observed to be much warmer than solar EUV-UV heating could maintain
(Bougher et al., 1993). The "heat island" formerly simulated near LT $=20$ for the MTGCM (benchmark) case is now located near mid-night, with the coldest exobase temperatures $(152 \mathrm{~K})$ now predicted near LT $=22$. This modification of the exobase thermal distribution is due in part to the impact of the semi-diurnal tides upon the global circulation. Convergence is now observed to occur near mid-night and along the morning terminator. Polar night (LT $=0-8$ ) temperatures (northward of $60 \mathrm{~N}$ ) are much warmer than before, a symptom of enhanced horizontal advection into this polar night region. Clearly, the Martian thermospheric dynamics is strongly modified as a result of dust-driven semi-diurnal tides. The corresponding exobase thermal structure is modestly changed, especially in the Northern polar night region.

Figure 4(b) illustrates MTGCM (dusty) equatorial temperatures over all local times from 100 to $300 \mathrm{~km}$. Again, nightside contours do not extend up to $300 \mathrm{~km}$. However, the premidnight region is predicted to be the coldest. The warmest temperatures $(363 \mathrm{~K})$ occur near $\mathrm{LT}=15$, in response to the semi-diurnal tides. The diurnal thermal contrast at exobase heights is now nearly $200 \mathrm{~K}$, with a nightside temperature minimum of about $160 \mathrm{~K}$. Again, a Venus-like nightside very cold thermosphere (i.e. cryosphere) is not predicted; yet, the day-night contrast is rather large, due to the role of tides in alterring the global circulation system. Overall, temperatures are fairly isothermal everywhere above 210-220 $\mathrm{km}$. It is of particular importance that the mesopause region (over 100-120 km) is strongly influenced by the dust-driven semi-diurnal tides. A wave \#2 signature is clearly seen in this region, with temperature minima $(92 \mathrm{~K})$ and maxima $(150 \mathrm{~K})$ centered around $110 \mathrm{~km}$. The phases of these tides clearly shift westward with increasing height, in accord with vertical propagation. However, this shifting ceases at $\sim 130 \mathrm{~km}$ where dissipation and molecular thermal conduction serve to damp the tides and minimize their effects at higher altitudes. It appears that a dusty lower atmosphere gives rise to substantial semi-diurnal tides that greatly impact the Mars mesopause region (100-120 km). However, at higher altitudes, these tidal effects are partially damped out, leaving modest changes in the exobase thermal structure.

Corresponding MTGCM (dusty) neutral and electron densities are not plotted. However, it is clear that warmer dayside temperatures and larger scale heights cause atomic $\mathrm{O}$ to exceed $\mathrm{CO}_{2}$ at slightly higher altitudes $(240-260 \mathrm{~km})$ than for the MTGCM benchmark case. Oxygen mixing ratios also display a rather flat distribution across the dayside, suggesting that $\mathrm{O}$-atom transport has been alterred. The electron density peak height at $60^{\circ} \mathrm{SZA}$ is now predicted to rise to about $140 \mathrm{~km}$. This increase of $\sim 5 \mathrm{~km}$ over the MTGCM benchmark case is largely a reflection of atmospheric inflation due to increased lower atmosphere aerosol heating (see Bougher et al., 1997). The Mariner 9 nomimal mission witnessed the rise of ionospheric peak heights by as much as 20 km (Zhang et al., 1990). Such a massive global dust storm may not recur each Martian year; however, even moderate dust levels can have a noticable impact on the Mars upper atmosphere. Planet-B NMS measurements of densities and inferred temperatures above $150 \mathrm{~km}$ should be sufficient to identify dust-induced impacts on the Martian upper atmosphere. 


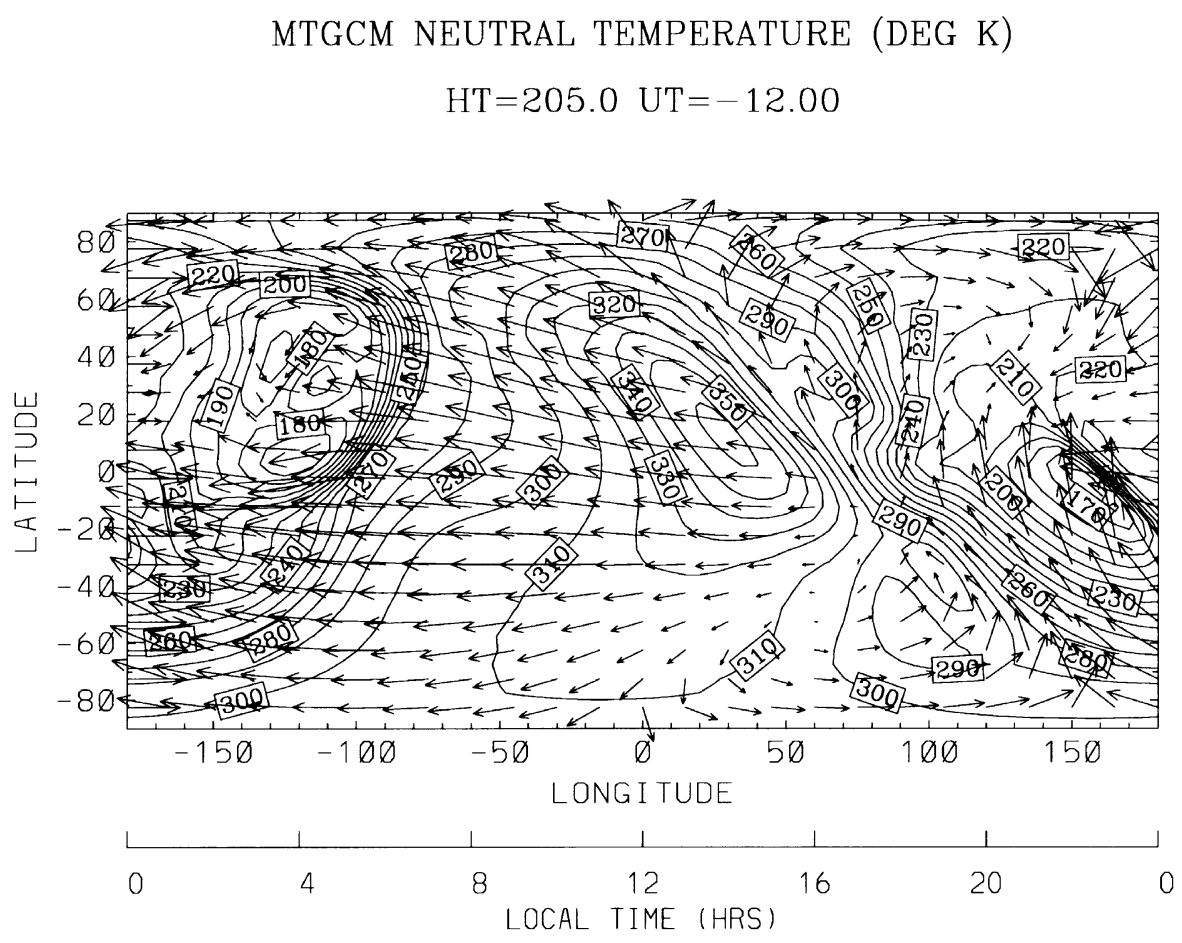

(a)

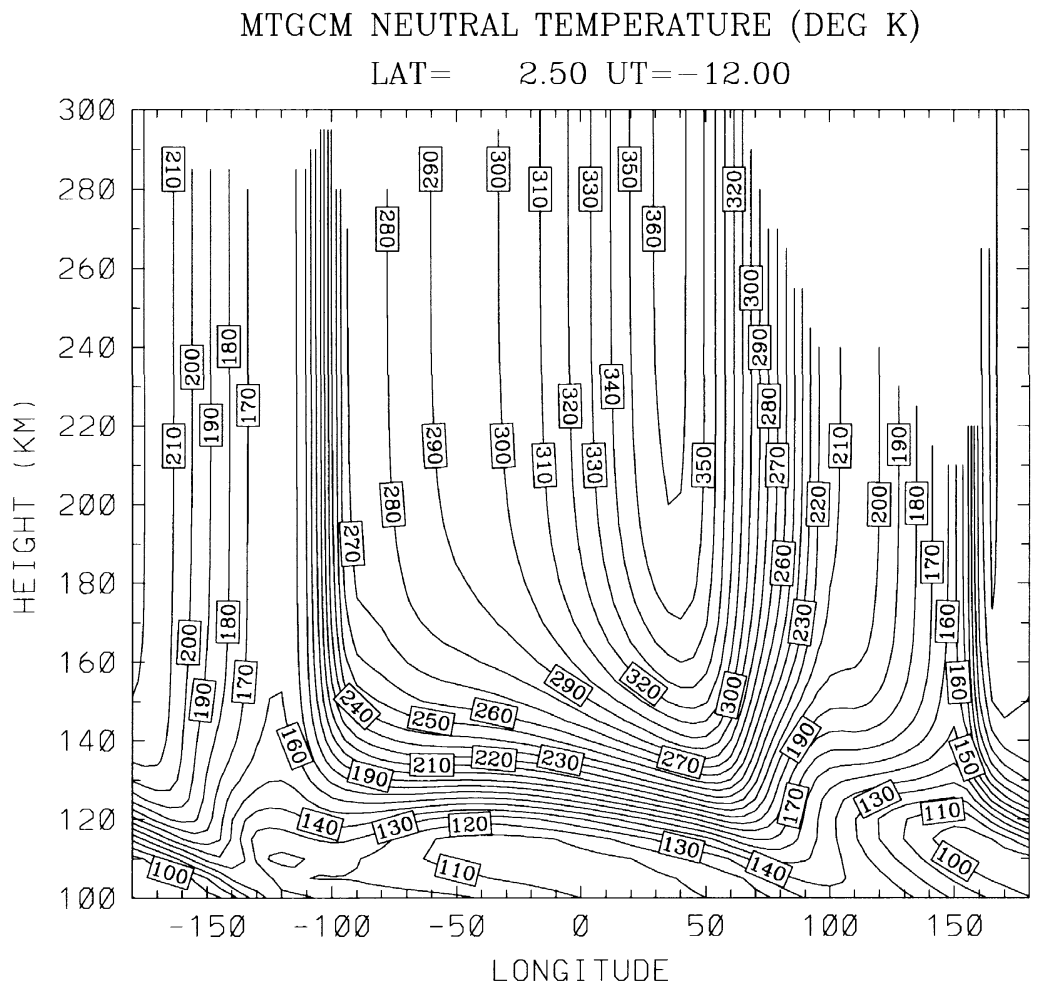

(b)

Fig. 4. Planet-B MOI MTGCM simulation (F10.7 = 102 at Mars; with dust): (a) Constant altitude slice (latitude vs. longitude or local time) at the exobase $(\sim 205 \mathrm{~km})$ for $T$ (total temperature) $+(u, v)$ (horizontal winds). Temperature extremes range from 151.6 to $362.5 \mathrm{~K}$. The length of the maximum arrow represents $400 \mathrm{~m} \mathrm{sec}^{-1}$. (b) $2.5 \mathrm{~N}$ latitude slice of total temperature over $100-300 \mathrm{~km}$, with extremes ranging from 92.3 to $362.9 \mathrm{~K}$. 


\section{Implications for MHD Modeling}

It has been shown that the density profiles of ions measured by the RPA on Viking (Hanson et al., 1977) cannot be reproduced without imposing a loss process at high altitudes (e.g. Chen et al., 1978; Shinagawa and Cravens, 1989; Fox, 1993). Debate also continues as to the appropriate electron temperatures to use at high altitudes for model simulations (Hanson and Mantas, 1988; Fox, 1993). The presently favored explanation for this loss process for maintaining observed dayside electron (and $\mathrm{O}^{+}$and $\mathrm{O}_{2}^{+}$) densities above $200 \mathrm{~km}$ is large-scale horizontal plasma convection. Such convection may occur as a result of an induced magnetic field at Mars (magnetic flux is supplied to the ionosphere from the magnetosheath region), and a corresponding solar-wind induced convection pattern (e.g. Shinagawa and Cravens, 1989). In this case, the Mars intrinsic magnetic field should be negligible. However, when the solar wind dynamic pressure exceeds the ionospheric thermal pressure, too much dayside ion loss is predicted to occur which drastically reduces the electron density (see Shinagawa and Cravens, 1989; Shinagawa, 1998, this issue). Therefore, a "moderate" convection driven in a weak Mars intrinsic magnetic field might also be a possibility. Finally, a small Mars intrinsic magnetic field may exist and a moderate magnetospheric convection flow might be present (Bauer and Hartle, 1973). These schemes all presume that dayside ion loss should be important above about $200 \mathrm{~km}$, thereby reducing $\mathrm{O}^{+}$and $\mathrm{O}_{2}^{+}$densities toward observed values. Photochemical equilibrium may still be a reasonable assumption for dayside ion densities below $\sim 200 \mathrm{~km}$ (Shinagawa and Cravens, 1989). Clearly, the magnetic field is the most sensitive ionospheric indicator of the Mars solar-wind interaction. Planet-B magnetometer measurements are essential to discriminate among these possibilities.

The MTGCM simulations above for photochemical ions serve as a starting point for detailed MHD calculations that can be made for various magnetic conditions at Mars. Dayside ionospheric conditions below $\sim 200 \mathrm{~km}$ may indeed reflect photochemical equilibrium behavior, especially during solar maximum conditions when the ionospheric pressure is greatest and the Mars ionosphere is likely unmagnetized (Zhang and Luhmann, 1992). However, the MTGCM code has no capability to self-consistently solve for solar-wind induced plasma convection. In the future, the MTGCM can be configured to incorporate divergence (transport) terms in the ion continuity equations, provided that ion drift velocities are supplied. Planet-B measurements will be made of ion drift velocities that can be used for this purpose. This will permit MTGCM nightside ion densities to be simulated owing to plasma transport about the globe.

\section{Conclusions and Future Work}

MTGCM thermosphere-ionosphere predictions appropriate for Planet-B Mars orbit insertion are made, both for solar EUV-UV forcing alone and also incorporating dust-driven forcings estimated for a moderately dusty lower atmosphere. It is clear that Mars conditions in early October 1999 should be similar to those experienced by Mariner 6-7 in 1969 . Accordingly, our predictions for dayside and nightside temperatures and winds show features reminiscent of previous
MTGCM simulations for Mariner 6-7. Dayside exobase temperatures near the equator rise to $310 \mathrm{~K}$; nightside values drop to 180-190 K. No nightside "cryosphere" of the type observed at Venus is presently predicted for Mars. The modeled electron density peak of $1.4 \times 10^{5} \mathrm{~cm}^{-3}$ is seen at 135 $\mathrm{km}$ at $60^{\circ} \mathrm{SZA}$. The impact of a dusty lower atmosphere is clearly felt in the Mars thermosphere, especially in the region of 100-120 km where dust-driven semi-diurnal tides manifest a strong perturbation upon otherwise solar EUVUV-IR driven temperatures and densities. Above $130 \mathrm{~km}$, partial damping of these tides occurs, leaving the exobase winds and temperatures only moderately changed. Mars ionospheric peak heights are certainly raised during dusty conditions as a result of atmospheric inflation arising from aerosol heating. Mid-afternoon and polar night exobase temperatures are also warmed considerably, owing to the effect of semi-diurnal tides upon the global thermospheric wind system. Planet-B NMS measurements of densities and inferred temperatures above $150 \mathrm{~km}$ should be sufficient to identify dust-induced impacts on the Martian upper atmosphere. The possibilities presented above, bracketed by a dust-free and dusty $(\tau=1.0)$ lower atmosphere case, provide a starting point for Planet-B data analysis.

Future planned improvements to the MTGCM thermosphere-ionosphere code include: (1) $\mathrm{O}^{+}$ambipolar diffusion, (2) $\mathrm{NO}_{x}$ neutral-ion chemistry, (3) the use of Tobiska (1991) solar EUV fluxes, and (4) the addition of an ion transport term to the ion continuity equations once ion drift velocities are available from Planet-B measurements or appropriate MHD model simulations. In addition, it is recognized that the present scheme utilized to couple the lower (Ames GCM) and upper (MTGCM) atmosphere models is in need of refinement. Specifically, tidal wave impacts will surely be modified by gravity wave interactions and eddy diffusion. This suggests the use of a more sophisticated coupling scheme that can incorporate various scales of waves that pass between the lower and upper Mars atmospheres, and simulate their interaction with the mean flow.

Most importantly, the MTGCM three-dimensional model will be maintained for community and local use, exercised systematically, and its results dispersed to various Planet-B teams as needed. MTGCM runs will be conducted for specific inputs appropriate to relevant Planet-B datasets. Postprocessing tools will also be supplied to each investigator to study MTGCM output for specific model simulations requested. This proposed facility approach to MTGCM model validation is analogous to that presently operational for the terrestrial TIGCM at NCAR, enabling TIGCM output to be used worldwide by various investigators. In a similar way, the MTGCM will support the scientific tasks planned by the Planet-B project for study of the Mars-solar wind interaction and the evolution of the Martian atmosphere over time. Planet-B collaborative studies making use of MTGCM outputs are now under discussion.

Acknowledgments. We are grateful to Dr. A. F. Nagy for encouraging the research outlined above. In addition, one of us (SWB) wishes to acknowledge the NASA Ames GCM modeling group and their contribution to MGCM-MTGCM coupling studies that have given rise to our estimates for dust impacts on the Mars thermosphereionosphere. 


\section{References}

Barth, C. A., A. I. F. Stewart, S. W. Bougher, D. M. Hunten, S. A. Bauer, and A. F. Nagy, Mars, Ch. 5.7: Aeronomy of the Current Martian Atmosphere, pp. 1054-1089, Univ. of Arizona Press, 1992.

Bauer, S. J. and R. E. Hartle, On the extent of the Mars ionosphere, J. Geophys. Res., 78, 3169, 1973.

Bougher, S. W., Comparative thermospheres: Venus and Mars, Adv. Space Res., 15, 21-45, 1995.

Bougher, S. W. and J. L. Fox, The Ancient Mars Thermosphere, Workshop on Evolution of Mars Volatiles, LPI Technical Report \#96-01, Part 1, pp. 5-6, 1996.

Bougher, S. W., R. E. Dickinson, R. G. Roble, and E. C. Ridley, Mars thermospheric general circulation model: Calculations for the arrival of Phobos at Mars, Geophys. Res. Lett., 15, 1511-1514, 1988.

Bougher, S. W., R. G. Roble, E. C. Ridley, and R. E. Dickinson, The Mars thermosphere II. General circulation with coupled dynamics and composition, J. Geophys. Res., 95, 14811-14827, 1990.

Bougher, S. W., E. C. Ridley, C. G. Fesen, and R. W. Zurek, Mars mesosphere and thermosphere coupling: Semidiurnal tides, J. Geophys. Res., 98, 3281-3295, 1993.

Bougher, S. W., D. M. Hunten, and R. G. Roble, $\mathrm{CO}_{2}$ cooling in terrestrial planet thermospheres, J. Geophys. Res., 99, 14609-14622, 1994.

Bougher, S. W., J. M. Murphy, and R. M. Haberle, Dust Storm Impacts on the Mars Upper Atmosphere, Adv. Space Res., 19, 1255-1260, 1997.

Chen, R. H., T. E. Cravens, and A. F. Nagy, The Martian ionosphere in light of Viking observations, J. Geophys. Res., 83, 3871, 1978.

Colin, L., Encounter with Venus, Science, 203, 743-745, 1979.

Fox, J. L., The production and escape of nitrogen atoms on Mars, J. Geophys. Res., 98, 3297, 1993.

Fox, J. L. and A. Dalgarno, Ionization, luminosity, and heating of the upper atmosphere of Mars, J. Geophys. Res., 84, 7315-7331, 1979.

Fox, J. L., P. Zhou, and S. W. Bougher, The Martian Thermosphere/Ionosphere at High and Low Solar Activities, Adv. Space Res., 17, 203-218, 1995.

Hanson, W. B. and G. P. Mantas, Viking electron temperature measurements: Evidence for a magnetic field in the Martian atmosphere, J. Geophys. Res., 93, 7538, 1988.

Hanson, W. B., S. Sanatani, and D. R. Zucarro, The Martian ionosphere as observed by the Viking retarding potential analyzers, J. Geophys. Res., 82, 4351-4363, 1977.

Joselyn, J. A., J. B. Anderson, H. Coffey, K. Harvey, D. Hathaway, G. Heckman, E. Hildner, W. Mende, K. Schatten, R. Thompson, A. W. P. Thomson, and O. R. White, Panel achieves consensus prediction of solar cycle 23, EOS Trans., American Geophysical Union, 78, 205-212, 1997.

Krasnopolsky, V. A., Solar cycle variations of the hydrogen escape rate and the CO mixing ratio on Mars, Icarus, 101, 33-41, 1993.

Murphy, J. R., J. B. Pollack, R. M. Haberle, C. B. Leovy, O. B. Toon, and J. Schaeffer, Three-dimensional numerical simulation of Martian global dust storms, J. Geophys. Res., 100, 26357-26376, 1995.

Nier, A. O. and M. B. McElroy, Composition and structure of Mars upper atmosphere: Results from the neutral mass spectrometers on Viking 1 and 2, J. Geophys. Res., 82, 4341-4349, 1977.

Pollack, J. B., R. M. Haberle, J. Schaeffer, and H. Lee, Simulations of the general circulation of the Martian atmosphere: 1. Polar processes, J. Geophys. Res., 95, 1447-1473, 1990.

Roble, R. G., E. C. Ridley, A. D. Richmond, and R. E. Dickinson, A coupled thermosphere-ionosphere general circulation model, Geophys. Res. Lett., 15, 1325-1328, 1988.

Rohrbaugh, R. P., J. S. Nisbet, E. Bleuler, and J. R. Herman, The effects of energetically produced $\mathrm{O}_{2}^{+}$on the ion temperature of the Martian thermosphere, J. Geophys. Res., 84, 3327, 1979.

Schatten, K. H. and W. D. Pesnell, An early solar dynamo prediction: Cycle 23-Cycle 22, Geophys. Res. Lett., 20, 2275-2278, 1993.

Schofield, J. T., D. Crisp, J. R. Barnes, R. Haberle, J. A. Magalhaes, J. R. Murphy, A. Seiff, C. LaBraw, and G. R. Wilson, Preliminary results from the Pathfinder Atmospheric Structure Investigation/Meteorology Experiment (ASI/MET), Science, 278, 1752-1758, 1997.

Seiff, A. and D. B. Kirk, Structure of the atmosphere of Mars in summer in mid-latitudes, J. Geophys. Res., 82, 4364-4378, 1977.

Shinagawa, H. and T. E. Cravens, A one-dimensional multispecies magnetohydrodynamic model of the dayside ionosphere of Mars, J. Geophys. Res., 94, 6506-6516, 1989.

Stewart, A. I. F., Revised time dependent model of the Martian atmosphere for use in orbit lifetime and sustenance studies, LASP-JPL Internal Rep., PO\# NQ-802429, Jet Propulsion Lab., Pasadena CA., March, 1987.

Stewart, A. I. and W. B. Hanson, Mars' upper atmosphere: Mean and variations, Adv. Space Res., 2, 87-101, 1982.

Stewart, A. I., C. A. Barth, C. W. Hord, and A. L. Lane, Mariner 9 ultraviolet spectrometer experiment: Structure of Mars upper atmosphere, Icarus, 17, 469-474, 1972.

Stewart, A. I. F., M. J. Alexander, R. R. Meier, L. J. Paxton, S. W. Bougher, and C. G. Fesen, Atomic oxygen in the Martian thermosphere, J. Geophys. Res., 97, 91-102, 1992.

Tobiska, W. K., Revised solar extreme ultraviolet flux model, J. Atmos. Terr. Phys., 53, 1005-1018, 1991.

Torr, M. R. and D. G. Torr, Ionization frequencies for solar cycle 21: Revised, J. Geophys. Res., 90, 6675-6678, 1985.

Torr, M. R., D. G. Torr, R. A. Ong, and H. E. Hinteregger, Ionization frequencies for major thermospheric constituents as a function of solar cycle 21, Geophys. Res. Lett., 6, 771-774, 1979.

Torr, M. R., D. G. Torr, and H. E. Hinteregger, Solar flux variability in the Schumann-Runge continuum as a function of solar cycle 21, J. Geophys. Res., 85, 6063-6068, 1980.

Yamamoto, T. and K. Tsuruda, The Planet-B mission, Earth Planets Space, 50, this issue, 175-181, 1998.

Zhang, M. H. G. and J. G. Luhmann, Comparisons of peak ionosphere pressures at Mars and Venus with incident solar wind dynamic pressure, J. Geophys. Res., 97, 1017-1025, 1992.

Zhang, M. H. G., J. G. Luhmann, A. J. Kliore, and J. Kim, A post-Pioneer Venus Reassessment of the Martian dayside ionosphere as observed by radio occultation methods, J. Geophys. Res., 95, 14829-14839, 1990.

S. W. Bougher (e-mail: sbougher@lpl.arizona.edu) and H. Shinagawa (e-mail: sinagawa@stelab.nagoya-u.ac.jp) 